ACCEPTED MANUSCRIPT

\title{
Nanoscale junctions for single molecule electronics fabricated using bilayer nanoimprint lithography combined with feedback controlled electromigration
}

To cite this article before publication: Alex Gee et al 2019 Nanotechnology in press https://doi.org/10.1088/1361-6528/ab6473

\section{Manuscript version: Accepted Manuscript}

Accepted Manuscript is "the version of the article accepted for publication including all changes made as a result of the peer review process, and which may also include the addition to the article by IOP Publishing of a header, an article ID, a cover sheet and/or an 'Accepted Manuscript' watermark, but excluding any other editing, typesetting or other changes made by IOP Publishing and/or its licensors"

This Accepted Manuscript is (C) 2019 IOP Publishing Ltd.

During the embargo period (the 12 month period from the publication of the Version of Record of this article), the Accepted Manuscript is fully protected by copyright and cannot be reused or reposted elsewhere.

As the Version of Record of this article is going to be / has been published on a subscription basis, this Accepted Manuscript is available for reuse under a CC BY-NC-ND 3.0 licence after the 12 month embargo period.

After the embargo period, everyone is permitted to use copy and redistribute this article for non-commercial purposes only, provided that they adhere to all the terms of the licence https://creativecommons.org/licences/by-nc-nd/3.0

Although reasonable endeavours have been taken to obtain all necessary permissions from third parties to include their copyrighted content within this article, their full citation and copyright line may not be present in this Accepted Manuscript version. Before using any content from this article, please refer to the Version of Record on IOPscience once published for full citation and copyright details, as permissions will likely be required. All third party content is fully copyright protected, unless specifically stated otherwise in the figure caption in the Version of Record.

View the article online for updates and enhancements. 


\title{
Nanoscale Junctions for Single Molecule Electronics Fabricated using Bilayer Nanoimprint Lithography combined with Feedback Controlled Electromigration
}

\author{
Alex Gee, ${ }^{a}$ Ayoub H Jaafar, ${ }^{a, b}$ Neil T Kemp ${ }^{a, *}$ \\ a Department of Physics and Mathematics, University of Hull, Hull, HU6 7RX, United Kingdom \\ ${ }^{\mathrm{b}}$ Physics Department, College of Science, University of Baghdad, Baghdad, 10071, Iraq \\ * Author to whom correspondence should be addressed. Electronic mail: N.Kemp@hull.ac.uk
}

Keywords: nanoimprint, lithography, molecular electronics, electromigration, bilayer resist, benzenethiol, molecular bridge, transition voltage spectroscopy

\section{Abstract}

Nanoimprint lithography (NIL) is a fast, simple and high throughput technique that allows fabrication of structures with nanometre precision features at low cost. We present an advanced bilayer nanoimprint lithography approach to fabricate four terminal nanojunction devices for use in single molecule electronic studies. In the first part of this work, we demonstrate a NIL lift-off process using a bilayer resist technique that negates problems associated with metal side-wall tearing during liftoff. In addition to precise nanoscale feature replication, we show that it is possible to imprint micronsized features while still maintaining a bilayer structure enabling an undercut resist structure to be formed. This is accomplished by choosing suitable imprint parameters as well as residual layer etching depth and development time. We then use a feedback controlled electromigration procedure, to produce room-temperature stable nanogap electrodes with sizes below $2 \mathrm{~nm}$. This approach facilitates the integration of molecules in stable, solid-state molecular electronic devices as demonstrated by incorporating benzenethiol as molecular bridges between the electrodes and characterizing its electronics properties through current-voltage measurements. The observation of molecular transport signatures, showing current suppression in the I-V behaviour at low voltage, which is then lifted at high voltage, signifying on- and off-resonant transport through molecular levels as a function of voltage, is confirmed in repeated I-V sweeps. The large conductance, symmetry of the I-V sweep and small value of the voltage minimum in Transition Voltage Spectroscopy indicates the bridging of the two benzenethiol molecules is by $\pi$-stacking.

\section{Introduction}

Since the initial ideas of using molecules to process signals, heralded by the molecular rectifier work of Aviram and Ratner[1] in 1974, scientists have steadily improved the understanding of these complex electrode-molecule systems and have developed better and more robust ways to integrate molecules into devices. In the last decade, molecular electronics has undergone a renaissance as a suite of new achievements has arisen. A single-molecule light emitting diode has been developed[2], single molecule rectification has reached levels of more than 200[3] and electrical control of the nuclear spin of a single molecule in a device has been achieved via the hyperfine Stark effect[4]. These 
achievements occurring because molecular electronics provides not only device miniaturization, but a route to the introduction of functional molecules with electronic, optical and mechanical properties tailored through chemical synthesis [5-9]. The future of molecular electronics is also bright. In recent years, traditional semiconductor devices have reached their scaling limit[10], and there is an increased need to find alternate ways to not only make electronic devices[11] but also explore new types of architectures[12,13] that non-traditional materials and methods of fabrication offer. However, many challenges in the field remain, especially at the single molecule electronic level, such as device variability, stability, improved size alignment between the "leads" and the "molecule" and upscaling from single to multiple device architectures to make computer-like systems.

Nanoimprint lithography (NIL) is a versatile nanofabrication technique that allows the replication of patterns with high throughput and resolution. In recent years it has experienced rapid development and uptake as a fabrication technique in both industrial and research environments [14-17]. One particular applicable field for NIL is molecular electronics. This field requires devices with highresolution sub-micron features and fabrication in large numbers in order to build-up statistically significant results.

In thermal NIL, a substrate is coated with resist which is heated above the glass transition temperature $\left(\mathrm{T}_{\mathrm{g}}\right)$ and then a stamp with the desired features is pressed into the coated substrate causing the resist to flow across the surface. After cooling, the substrate is mechanically separated leaving an impression of the stamp [18]. It currently has a demonstrated resolution of less than $10 \mathrm{~nm}$ [19].

NIL is commonly used as an etch mask for pattern transfer but it does not lend itself well to lift-off as the imprinting process always leaves the patterned resist with sidewall angles greater than $90^{\circ}$ as this is necessary to remove the stamp after imprinting. During metal deposition, material will adhere to the sidewalls and form a continuous film over features on the resist, making clean lift-off nearly impossible [20].

An alternative approach often used in lithography processes is the use of a lift-off resist (LOR) layer. This is a bottom resist layer that allows an undercut to be formed once the lithographic process has patterned the topmost layer. This process reduces the occurrence of an incomplete lift-off and tearing of the patterned metal films by making it very unlikely for a continuous metal film to exist between material patterned on the substrate and material deposited on the resist layer. We chose to apply this technique in NIL. Previously, it has been demonstrated that a bilayer resist coating can be imprinted without disturbing the bilayer structure for pillars [21]. However, this was demonstrated only for small pillars and lines with sizes between $20 \mathrm{~nm}$ and $600 \mathrm{~nm}$. Another technique exploiting the differing plasma etch resistance of polystyrene-polydimethylsiloxane (PS-PDMS) and polymethyl methacrylate (PMMA) has been shown to produce undercut resist structures with NIL [22]. However, the line quality of features produced using this technique may suffer due to the irregular nature of the top PS-PDMS layer after extended plasma exposure.

In addition to having high-quality lift-off, there is a need as well to be able to imprint nanometre as well as micrometre scale features with NIL. This is especially relevant in the case of electronic devices where nanosized features must be present alongside larger sized pads that are needed to make electrical connections with wire-bonding or a probe-station. Using standard NIL methods, this can be very difficult since large amounts of lateral resist flow occurs from imprinting the larger features, which can degrade or even make impossible the imprinting of the smaller nanoscale features. Using the above bi-layer approach, we explore this problem and show how to resolve this issue by choosing suitable imprint parameters and residual layer etching. 
Lastly, we demonstrate the success of the above technique in the fabrication of devices for molecular electronics. Molecular electronics needs reliable methods to fabricate gaps between electrodes, of order $2 \mathrm{~nm}$, and methods to integrate, in a reliable manner, single molecules that bridge between source and drain contacts. Electromigration is one such method to make very small gaps between electrodes [9,23-25]. Using our double layer NIL method we demonstrate the suitable fabrication of devices for electromigration and form molecular devices by incorporating benzenethiol as molecular bridges between the electrodes.

\section{Method}

The Master stamp used in this work was fabricated by standard electron beam lithography (EBL) and reactive ion etching (RIE) on a $100 \mathrm{~mm}$ diameter Si wafer and was cleaned prior to use with sonication in acetone, 2-propanol and water before being dried in nitrogen and baked prior to spin coating of the e-beam resist (AR-N 7520, Allresist). After the patterning process the stamp was coated with an antistick coating, perfluorodecyltrichlorosilane (FDTS), that aids stamp separation after imprinting. The $\mathrm{Si}$ wafer, shown in Figure 1 (a), was patterned in a square-like grid with $10 \times 12 \mathrm{~mm}$ separating each patterned area. Each patterned area contains a group of 15 imprintable devices arranged in a horizontal line, as shown in Figure 1 (b). The design of a single device consists of a cross with 4 arms that are used for 4-terminal electrical connections. In the centre of the cross the four arms meet and form a single, nanoscale, vertical line with dimensions of $100 \times 200 \mathrm{~nm}$, as shown in Figure 1 (c), and having two arms connected to either side. The nanoscale line is the main part of the device and is used to make the conducting channel for the electromigration process. The electromigration process occurs after the NIL process and is used to make the nanoscale junction, which consists of a very small airgap $(<2 \mathrm{~nm})$ between two metal electrodes.

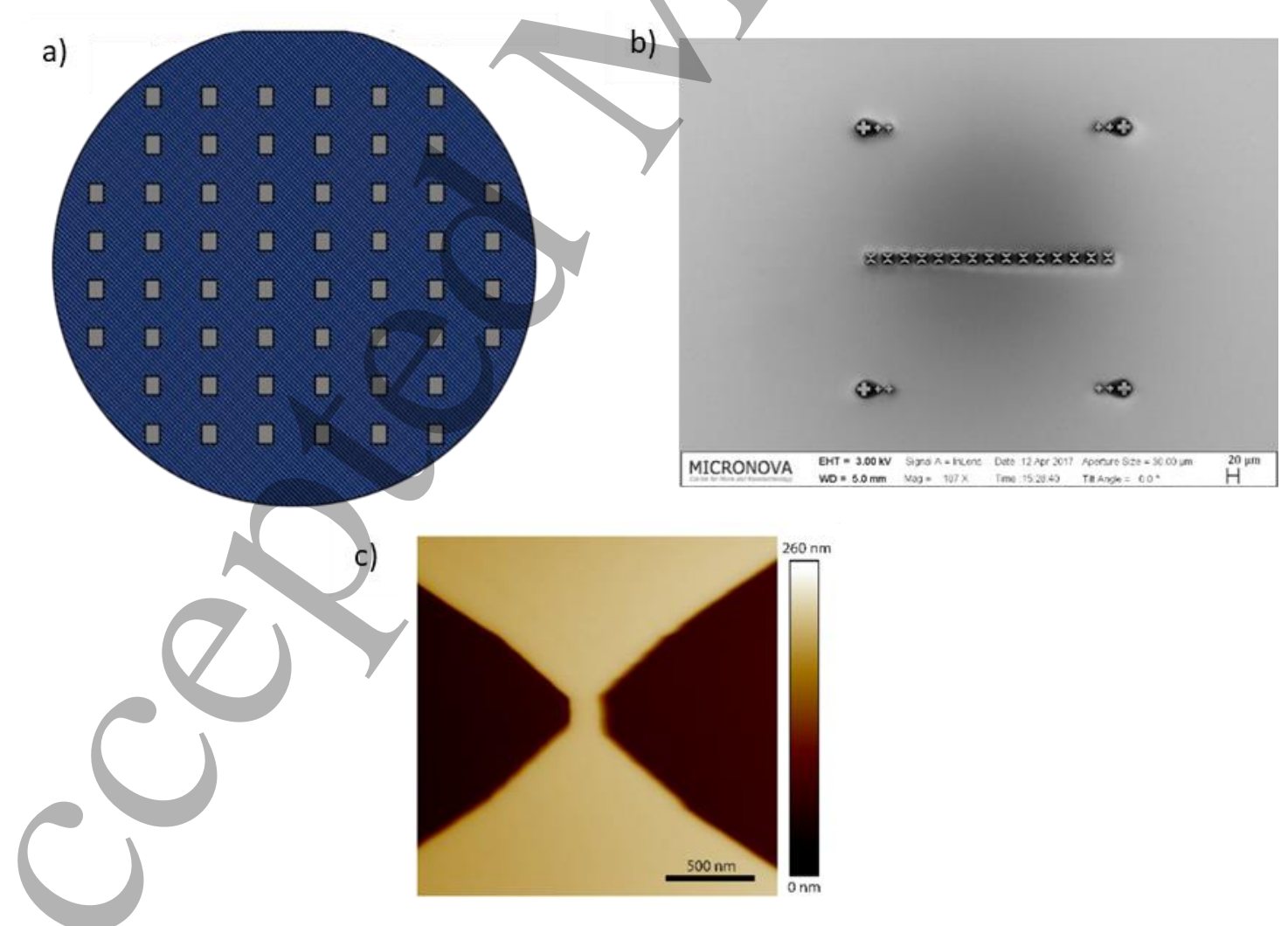

Figure 1. a) Stamp design consisting of 56 groups of 15 nanojunction (crosses) on a $100 \mathrm{~mm}$ diameter Si wafer fabricated using EBL and RIE. b) SEM image of one group of 15 nanojunctions showing marks for 
photolithography alignment. c) AFM height map of the stamp and showing the channel region (yellow) that is at the centre of a single cross and used to make a nanojunction by electromigration.

The NIL method used for copying the master pattern is shown in Figure 2 and consists of the following steps. Firstly, the bilayer resist was deposited onto a cleaned Si wafer with $1 \mu \mathrm{m}$ of thermally grown oxide. The bilayer consisted of polymethylglutarimide (PMGI) SF5 resist (MicroChem Corp), spincoated to give a thickness of $150 \mathrm{~nm}$ and baked at $190^{\circ} \mathrm{C}$. This was followed by a layer of $15 \mathrm{kPMMA}$ that was dissolved in chlorobenzene ( $8 \% \mathrm{wt}$.), spin coated to a thickness of $200 \mathrm{~nm}$ and baked at $160^{\circ} \mathrm{C}$. Imprinting was carried out by applying 2 bar pressure (used also for all test studies described in this work) once the set-point temperature was reached. Once the desired imprinting time was reached, the stamp was cooled whilst still maintaining pressure until the temperature had reached $90^{\circ} \mathrm{C}$. The stamp is separated by inserting a scalpel blade between the stamp and wafer and the residual PMMA is removed using low power oxygen plasma.

Spin coated films and residual layer thicknesses were characterised by AFM (Bruker Dimension Edge) and/or surface profilometry (Bruker DektakXT). A CNI v2.1 machine (NILT) was used to perform the imprinting and the process of coating the stamps and NIL was carried out in a class 1000 cleanroom. Metal deposition was carried out using e-beam evaporation in a system with base pressure of $1 \times 10^{-7}$ mbar (HHV Auto500). The deposition rate was maintained at $0.1 \AA / \mathrm{s}$ and the substrate temperature did not rise above $40^{\circ} \mathrm{C}$ to not influence the imprinted resist structure,

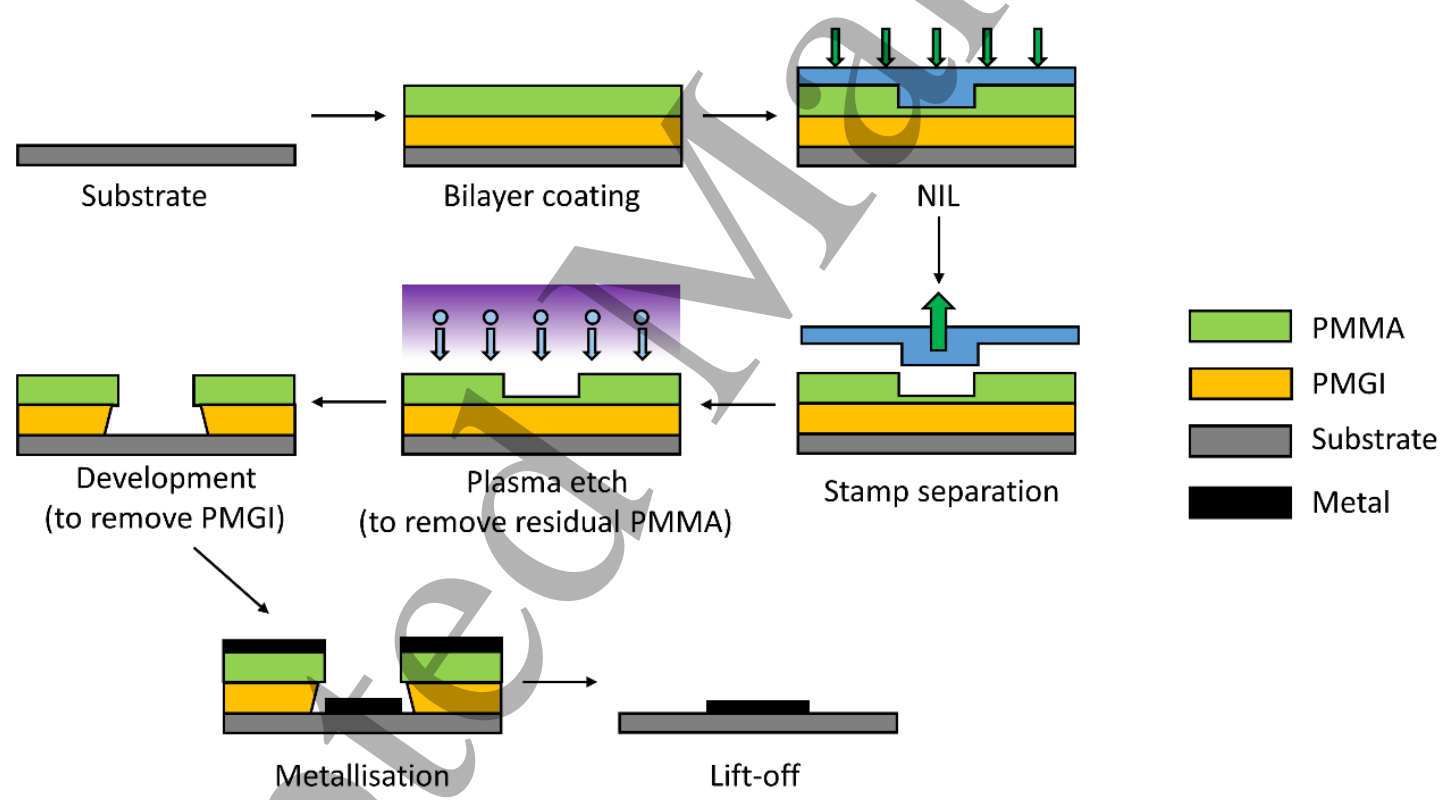

Figure 2. The Bilayer NIL process consists of spin-coating a substrate with a bilayer of PMGI/PMMA, imprinting and removal of the stamp. This is followed by plasma etching to remove residual PMMA resist, which then permits an undercut to be formed by selective removal of PMGI, allowing metallisation and a clean lift-off.

\section{Results and Discussion}

The results and discussion section is divided into three sections, covering i. Optimization of the bilayer nanoimprint lithography process for devices containing both nano and micron feature sizes, ii. The electromigration process and iii. Single molecule electronic devices. 


\section{i. Bilayer Nanoimprint Lithography for Devices containing Both Nano and Micron Feature Sizes}

In order to determine the correct imprint parameters several trial imprints were made across a range of temperatures $\left(140^{\circ} \mathrm{C}\right.$ to $\left.190^{\circ} \mathrm{C}\right)$ whilst keeping the resist material, film thickness and imprint pressure constant. As expected, higher temperatures resulted in faster imprints, as shown in Figure 3 (b). For process temperatures greater than $150^{\circ} \mathrm{C}$, very little change in imprinted depth was observed after 120 seconds, which signifies that the imprinting is complete in this time.

At the highest temperature, a reduction in the quality of the imprinted features was observed. Voids also appeared in the films when heated above $190^{\circ} \mathrm{C}$. It is possible that intermixing of the polymer bilayer may occur above this temperature resulting in localised disturbances of the film surface giving the appearance of voids. At $140^{\circ} \mathrm{C}$ and $150^{\circ} \mathrm{C}$, very slow imprinting occurred and the displaced resist was observed to flow upwards rather than laterally, producing raised regions. This can be seen in Figure 3 (a) as rings of defect free resist surrounding the imprinted regions[26]. (Note: when this occurred, the imprinted depth was measured from the undisturbed resist level away from the imprint.)

For imprints carried out at temperatures between $140^{\circ} \mathrm{C}$ and $170^{\circ} \mathrm{C}$, a reoccurrence of void defects in the resist film was also observed. Unlike those observed at temperatures above $190^{\circ} \mathrm{C}$, these defects likely occur as a result of the stamp bending around the stamp protrusions which are in the process of imprinting. The resulting small separation between the low viscosity resist surface and stamp as a result of this bending has been shown to result in a polymer thinning effect as a result of electrostatic interactions between the stamp and resist surface [27]. At $180^{\circ} \mathrm{C}$, only a very small number of these void defects occurred, usually located in proximity to dust particles present on the resist but not around the desired features. This is most likely because of reduced stamp bending as the polymer has a reduced viscosity at this higher temperature. Reducing foreign particles should totally eliminate the occurrence of this type of defect at this temperature and imprinting pressure.

a)
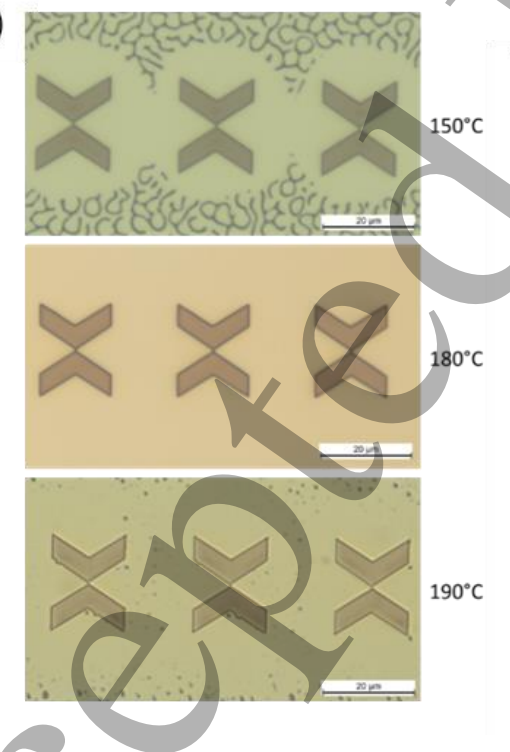

b)

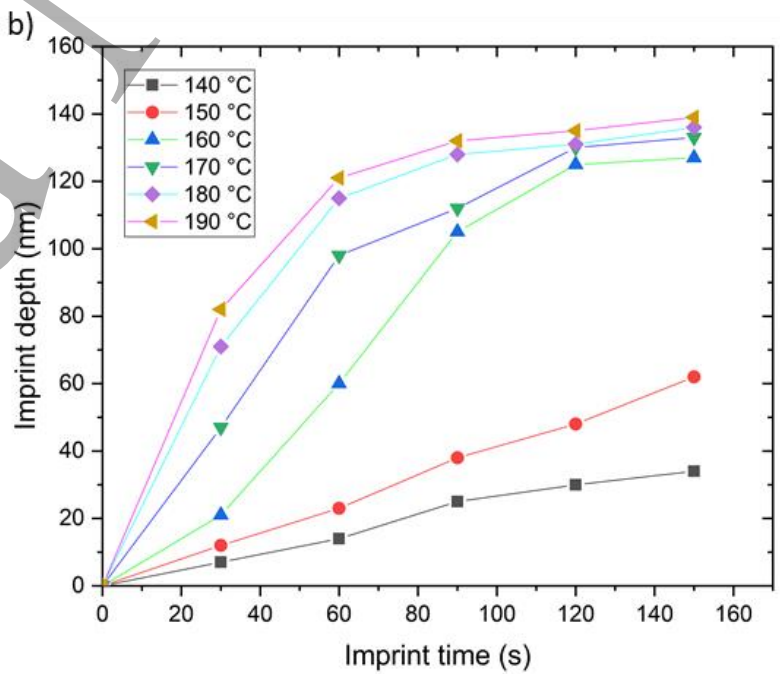

Figure 3. a) Imprinting of the nanojunction (Master) stamp into bilayer resist at increasing temperatures leads to the appearance of flow defects. Areas immediately surrounding the imprinted features appear well imprinted due to a squeeze flow of resist at $150^{\circ} \mathrm{C}$ while at $190^{\circ} \mathrm{C}$ voids appear. $180^{\circ} \mathrm{C}$ gives optimum imprinting behaviour. b) Imprinting depth measured at increasing temperature into $150 \mathrm{~nm}$ thick, 15k PMMA resist with constant applied pressure. The CNI imprinter was rapidly cooled to a temperature below $110^{\circ} \mathrm{C}$ in order to halt the imprinting process, however it is likely that some small amount of imprinting depth will occur during cooling. 
The bilayer NIL process produces an unwanted residual layer of PMMA $(20 \mathrm{~nm})$ after stamping that must be removed before the development and metallization processes. An isotropic oxygen plasma etch (Diener Zepto, 14 minutes) was used to remove this layer. As well as removing the residual polymer, the process can also broaden the imprinted features and for this reason, the duration of etching should be limited to preserve the size of the original design. The correct plasma etch duration was determined by an iterative process. For under-etching, either no development of the PMGI occurred as the residual PMMA layer completely blocks access of the developer solution, or islands of PMMA were observed, which can only be removed after excessive development time which then risks collapse of the bilayer structure.

The correct development time is also crucial to the process. If the development time is too short, remnant PMGI is present as shown by the yellow shadowing of the edges in Figure 4 (a), resulting in poor metallization and lift-off. If the development time is too long, the quality of the replication is poor as there is a partial collapse of the edges, as shown in Figure 4 (c). A development time of $20 \mathrm{~s}$ was found to be ideal for the thickness of the PMGI layer used, resulting in clean and crisp features, as shown in Figure 4 (b).

a)

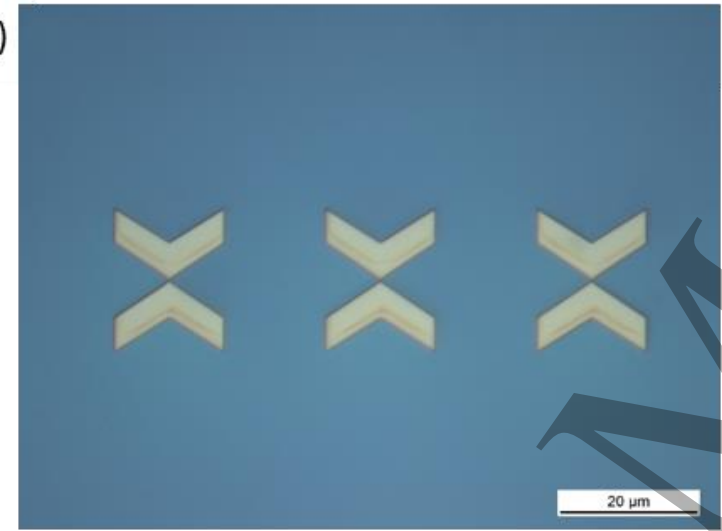

c)

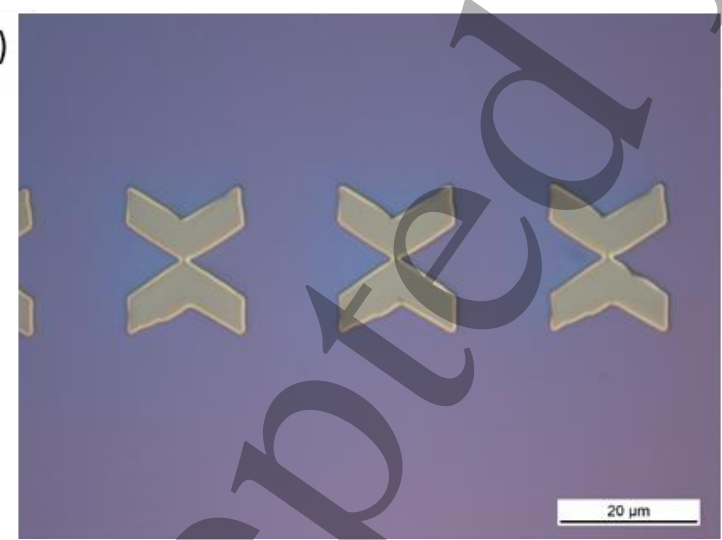

b)

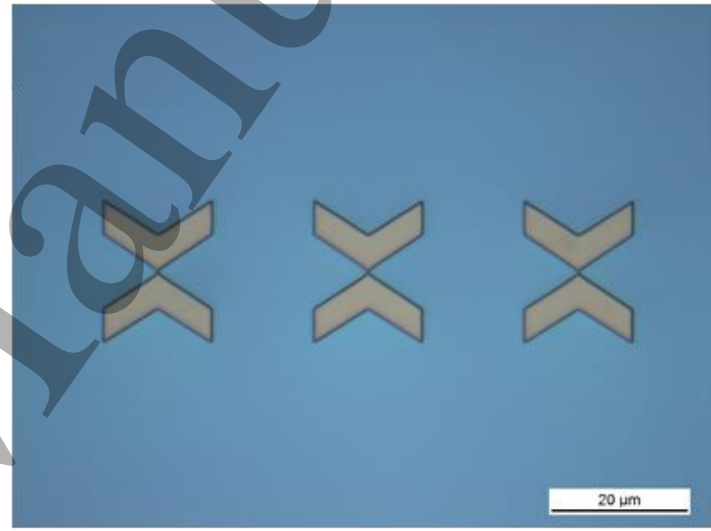

d)

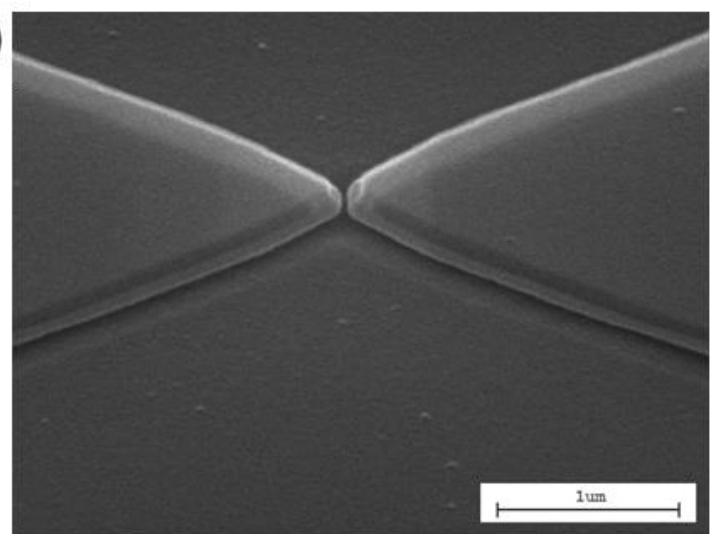

Figure 4. Increasing the length of development from a) $10 \mathrm{~s}$, b) $20 \mathrm{~s}$ and c) 30 s of the features in PMMA/PMGI bilayer resist after removal of the residual layer of resist through plasma etching. Imprint time d) SEM image of the nanojunction règion after 20s development.

After patterning of the resist by the NIL process, $20 \mathrm{~nm}$ of gold was deposited onto the wafer, followed by lift-off using acetone and then $\mathrm{n}$-methyl-2-pyrrolidone. Substrates were also gently cleaned using a low power (25W) oxygen plasma exposure for 10 minutes. In the case of the devices used for molecular electronics studies, a two-step lithography approach was used. The second lithography step involved a standard UV lithography process and was used to pattern large area and thick Al contact 
pads onto the ends of the four arms of the crosses in order to facilitate electrical connections. The completed devices, in this case, are shown in Figure 5.

Using this approach, and with periodic cleaning of the Master stamp using sonication in acetone and 2-propanol, we have been able to carry out the NIL process over 50 times with no degradation in the imprinted features making this process very attractive for producing large numbers of devices. We see no reason why this process cannot be continued many hundreds of times.
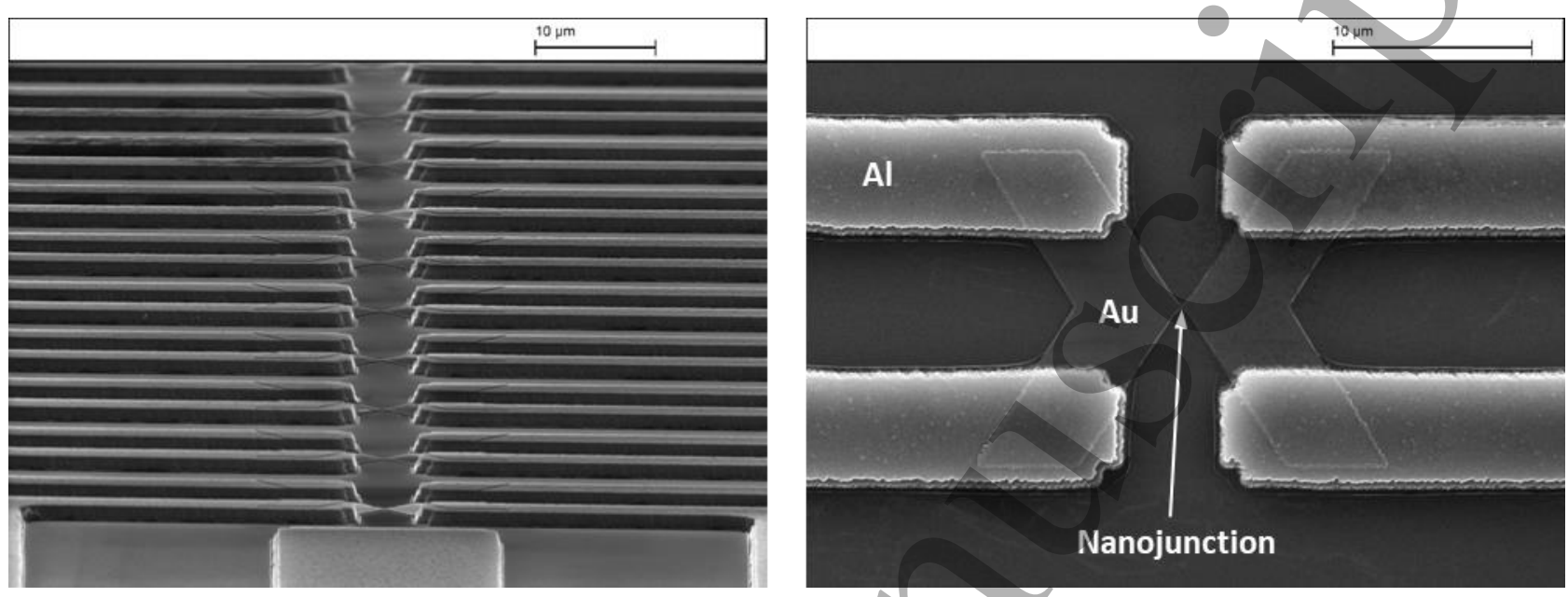

Figure 5. SEM images of a completed device consisting of 15 Au nanojunctions connected to thick Al contact pads.

\section{ii. Electromigration}

In this work, electromigration was used to make very small, nanosized gaps $(<2 \mathrm{~nm})$ for molecular electronics studies. Electromigration is a process in which a large current density is driven into a narrow and confined conducting channel $[28,29]$. The related electron wind of the current displaces atoms and defects, causing them to migrate. Once the current density flowing through the constriction surpasses the electromigration threshold (typically $>10^{8} \mathrm{Am}^{-2}$ for gold[30]) the resistance of the nanojunction increases as its cross-sectional area narrows due to material transport. This results in a thinning (or pinching) of the conduction channel in the lateral direction, and usually occurring at the midpoint of the channel where there is maximum heating or at desired position along the length of the channel, that can be determined by placing a notch. With continued application of a current density, usually imparted via voltage pulses, the pinching effect occurs until at some point, a single atom is present in the gap, as typically confirmed by the observation of quantized steps in the conductance[31]. With time, and or more application of pulses, a nanojunction typically forms, which generally consists of the formation of a sub- $2 \mathrm{~nm}$ gap between the two electrodes [23].

Thermal runaway is a problem/inherent with electromigrated break junctions [24,32]. This occurs because the nanojunction resistance increases as electromigration begins and the power being dissipated by the junction, in the form of heat, also increases. The rate of electromigration is increased with elevated temperature and a thermal runaway will most likely occur in the absence of any form of limiting mechanism [33,34]. Nanojunctions broken as a result of thermal runaway tend to have large gaps [35]. Previously reported nanoimprinted devices have been shown to produce large and uncontrollable gap sizes and in addition require heating of the device in order to increase the nanojunction resistance and increase material diffusion [36]. When used for molecular electronics studies this requirement poses the potential risk of thermal decomposition of any molecules present 
prior to breaking as well as also excluding the possibility of carrying out electromigration in experiments that study low temperature molecular phenomenon. In our system, this process is avoided by using a feedback voltage source (FVS). This circuit maintains a constant voltage across the nanojunction with the result that the power dissipated by the junction is reduced as electromigration progresses. Using a four terminal device allows us to compensate from resistance that arises as a result of the thin film interconnects on the device as well as contact resistance from the probes used to connect the device to equipment.

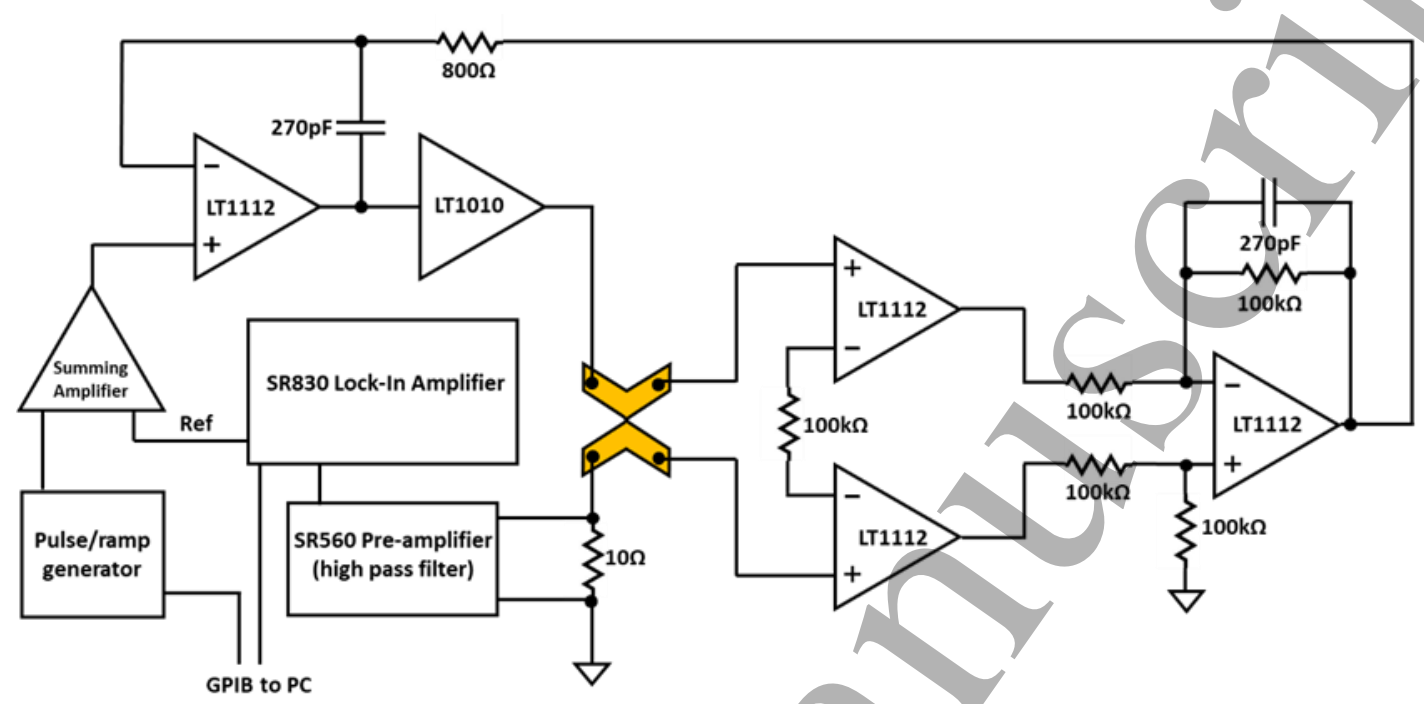

Figure 6. Schematic of the experimental setup including feedback voltage source used to carry out electromigration of the nanojunctions. The gold cross represents the nanojunction device

Figure 6 shows the schematic of the FVS. The voltage drop across the junction is measured by an additional pair of leads that connect to an instrumentation amplifier, the output of which is fed into the non-inverting input of the feedback op-amp. The circuit is stabilised by tuning the capacitance and resistance in the feedback stage such that an adequately fast response is obtained whilst preventing overshoot and oscillation when applying step changes in voltage, similar to the pulses used to carry out electromigration. It was found that adding capacitance across the feedback path of the instrumentation amplifier was also needed in order to provide a stable feedback mechanism without excessive overshoots and oscillation. In our setup, the resistance of the junction is monitored by a lock-in technique by measuring the voltage drop across a $10 \Omega$ resistor between the junction and ground. The lock-in excitation $(4 \mathrm{mV}, 1 \mathrm{kHz})$ and is added to the voltage pulses which drive the electromigration process ( $17 \%$ duty cycle, $17 \mathrm{~Hz}$ ). The setup is computer controlled to increase the voltage applied to the device incrementally per pulse (approximately $150 \mu \mathrm{Vs}^{-1}$ ) while continually monitoring the nanojunction resistance. As electromigration progresses we see transitions from normal diffusive conduction, to a quasi-ballistic regime as the nanojunction size reduces below the electron scattering length to a size of just a few atoms. At this point only a few transport channels contribute to the overall conduction of the device and in some runs we observe quantised conductance values just before the wire breaks to form nano-sized gap. A sudden jump in resistance indicates that a tunnelling gap has developed, after which the electromigration procedure is automatically halted by the program.

Figure 7 (a) shows the evolution of the electromigration process in the formation of a typical nanojunction. The resistance vs pulse voltage shows the transition from the conductive diffusive 
regime (high conductance) to the quasi-ballistic regime, with steps in the conductance in the vicinity of $G_{0}$ denoting a limited number of quantized conduction channels. Figure 7 (b) shows a typical AFM image of one junction after undergoing electromigration to form a nanogap. It is clear that a change in the junction dimensions has occurred. However, the actual gap size is well below the resolution of the instrument. We therefore rely on electrical measurements to determine the typical gap size. Figure 7 (c) shows a typical nonlinear current-voltage (I-V) characteristics fitted to the Simmons tunnelling model (equation 1 ) derived from $\mathrm{dl} / \mathrm{dV}$ measurements. Although we are not able to measure directly the barrier height or the junction area, these two parameters are quite robust and the fit is determined mainly by the gap size. Fitting over 10 such devices with $\varphi=1 \mathrm{eV}$, we find that the gap size is less than $2 \mathrm{~nm}$ confirming that electromigration can be carried out smoothly in devices fabricated using this bilayer technique.

a)

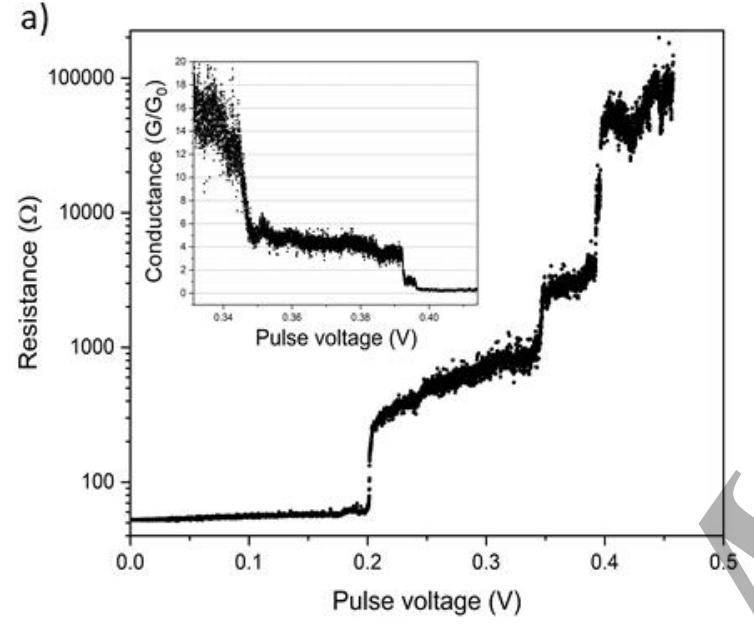

b)

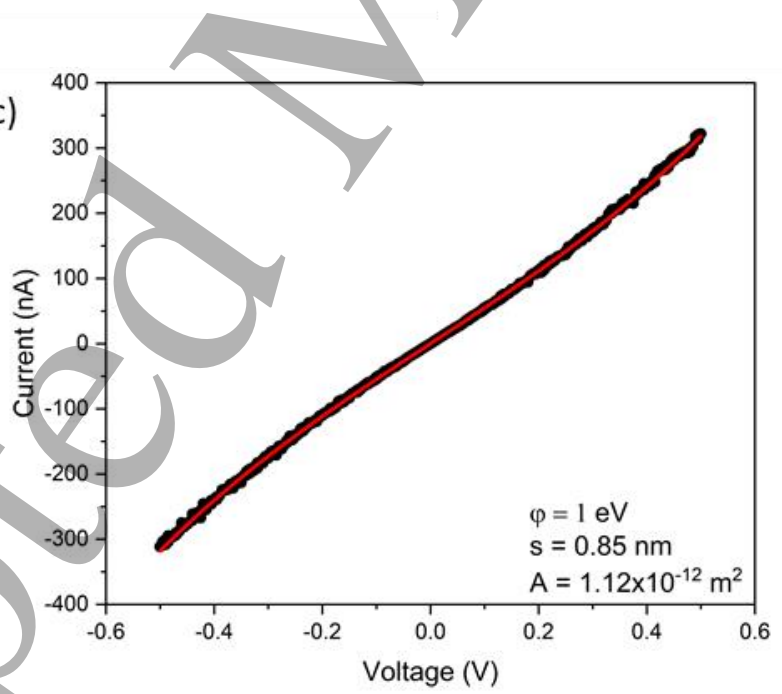

Figure 7. a) Evolution of the device resistance during the electromigration procedure. Resistance is measured between voltage pulses. Inset shows the conductance plotted in terms of the quantized conductance, $\mathrm{G}_{0}$, in the region from $20 G_{0}$ to before the transition into a tunnelling regime $\left(<1 G_{0}\right)$. b) AFM image of a nanojunction after electromigration has formed a nanogap. c) Representative I-V curve measured at room temperature after a nanogap has formed in two devices and showing a fit to the Simmons tunnelling model. 
The Simmons tunnelling model:

$$
I(V)=\frac{A e}{2 \pi h(\beta \Delta s)^{2}}\left\{\varphi \exp \left(\frac{4 \pi \beta \Delta s}{h} \sqrt{2 m} \sqrt{\varphi}\right)-(\varphi+e V) \exp \left(\frac{4 \pi \beta \Delta s}{h} \sqrt{2 m} \sqrt{\varphi+e V}\right)\right\}
$$

where $I$ is the current, $A$ is the junction area, $\Delta s$ is the gap size, $\varphi$ is the barrier height and $V$ is the applied voltage [37]. $\beta$ is a constant which can be approximated to unity in the voltage regime over which we make measurements.

\section{iii. Single Molecule Electronic Device}

As a proof of concept of our fabrication process, single molecule electronic devices were made containing diphenyl disulphide (DPDS). This compound consists of two phenyl rings connected via two thiol groups. It has been well documented that upon exposure to a gold surface the S-S bond of the DPDS molecule oxidizes to form benzenethiol (BT), which self-assemble onto the electrode surfaces [38-40]. The resulting BT monolayers present an ideal testbed for measuring signatures of molecular electronic transport due to the high conductivity of the delocalised $\pi$-orbitals on the phenyl rings. In addition, the versatility of this molecule allows for the construction of many types of molecular junction with differing functionalities though step-wise chemical synthesis with BT forming the initial anchoring unit of a molecular wire [41].

In a typical molecular transport experiment, the molecules are allowed to self-assemble onto the nanojunction and electromigration is used to form a nanogap. The increased temperature during the electromigration process is expected to increase the mobility of molecular species present in the vicinity of the junction area and increase the chance of a molecule moving into the nanogap once formed. An advantage is that the experiment can be performed in situ without the need to remove the device from the measurement setup, which would otherwise expose the clean nanogap to ambient contamination. We first oxygen plasma clean the unbroken nanojunction ( 15 minutes, $50 \mathrm{~W}$, $0.35 \mathrm{mbar}$ ) before removing them from the etching chamber and immediately immersing the device into a $0.04 \mathrm{mM}$ solution of DPDS in ethanol for 30 minutes. We then carry out the electromigration procedure and measure the I- $V$ characteristics of the device. Out of 10 electromigrated nanogaps exposed to DPDS, two measured devices displayed behaviour that was indicative of molecular transport i.e. current suppression at low voltage, due to off-resonant transport, and a lifting of the current suppression at higher voltage. In contrast, devices that contained no DPDS molecules exhibited the typical Simmons I-V curve that is shown in Figure 7 (c). Furthermore, at a voltage of 0.5 $\mathrm{V}$, empty junctions typically had a current of $300 \mathrm{nA}$ compared to $1 \mu \mathrm{A}$ for a junction containing exposed to DPDS, as shown in Figure 8 (a). 

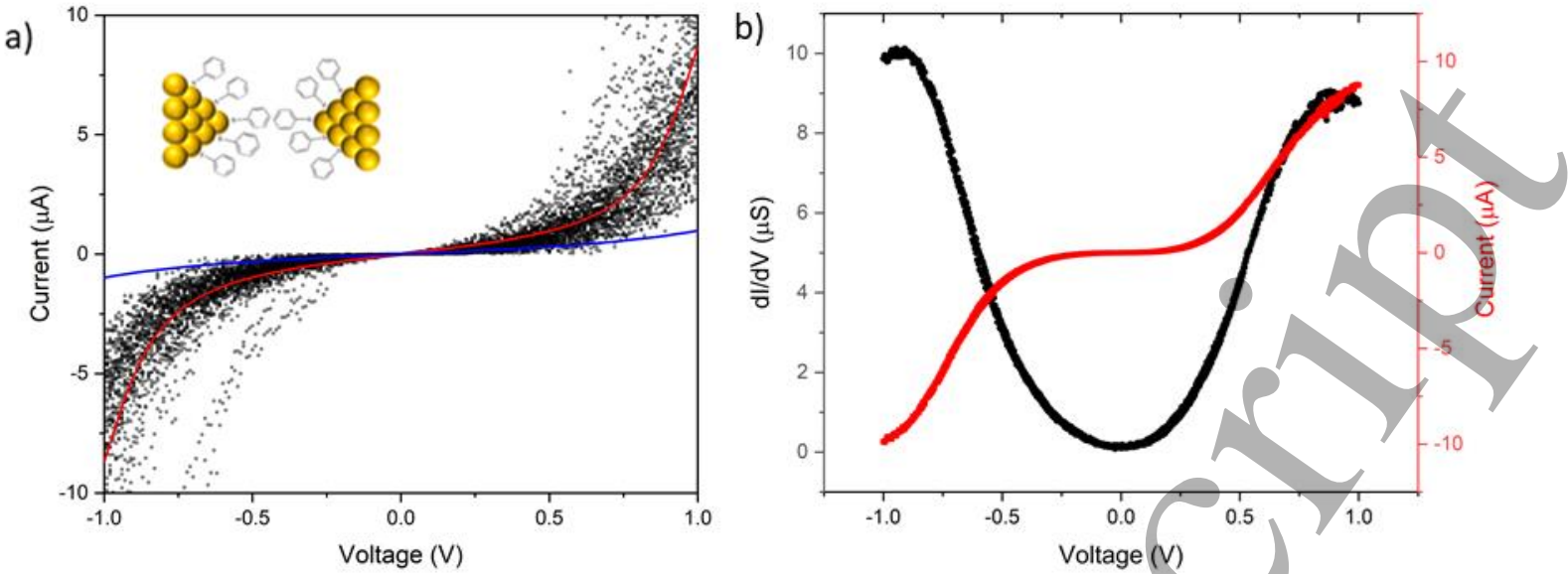

c)
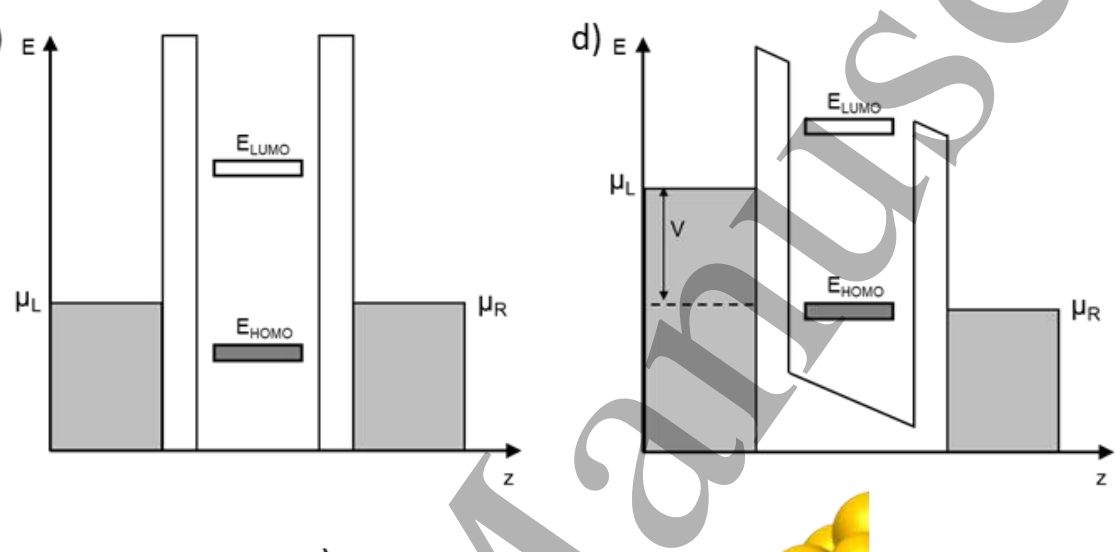

e)

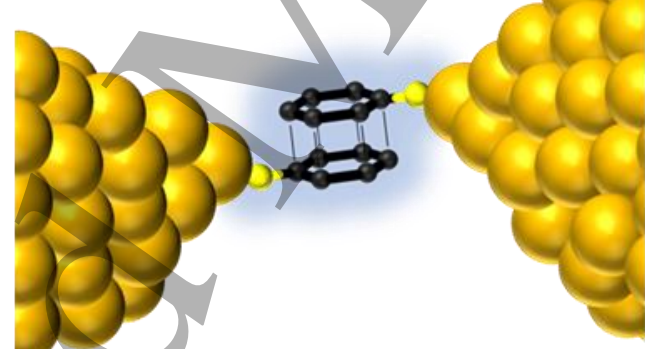

Figure 8. a) Repeated I-V measurements made on a DPDS electromigrated nanogap made over a 12 hrs, (lockin technique $4 \mathrm{mV}$ at $1447 \mathrm{~Hz}$ ). Current is extracted from dl/dV measurement. Red curve is a fit to the resonant tunnelling model (equation 2) with $\Gamma s, \mathrm{D}=34 \mathrm{meV}, \varepsilon_{0}=-0.49 \mathrm{~V}$. The blue curve, showing the lack of a good fit using the Simmons model $\left(\varphi=1 \mathrm{eV}, \mathrm{s}=0.85 \mathrm{~nm}, \mathrm{~A}=1.12 \times 10^{-12} \mathrm{~m}^{2}\right)$ indicates that the junction is not an empty tunnel gap. Inset - a schematic of a possible DT configurations within the nanogap. b) Selected single I-V curve (red) and $\mathrm{dl} / \mathrm{dV}$ (black) from another junction showing similar current suppression c) Schematic of molecular conduction through a gold-BT-BT-gold junction at low voltage where the current is suppressed due to offresonant transport. d) With application of a sufficiently high voltage, the levels shift bringing a molecular level into resonance, lifting the current suppression. e) Expected molecular configuration of the nanojunction consisting of overlapping $\pi$ orbitals from stacked benzene rings that are thiol bonded to opposite electrodes.

Figure 8 (a) shows the I-V behaviour of a device exposed to DPDS, electromigrated and then measured continuously over a $12 \mathrm{hr}$ period. The I-V curves show some variation, mostly liked due to minor movement of the molecules due to thermal effects, but the current suppression expected at low 
voltage is clearly visible. We fit the data with again with the Simmons model as well as a single level resonant tunnelling model (equation 2 ):

$$
I(V)=\frac{4 G_{0}}{e} \frac{\Gamma_{S} \Gamma_{D}}{\Gamma_{S}+\Gamma_{D}}\left[\arctan \left[\frac{\varepsilon_{0}+e V / 2}{\Gamma_{S}+\Gamma_{D}}\right]-\arctan \left[\frac{\varepsilon_{0}-e V / 2}{\Gamma_{S}+\Gamma_{D}}\right]\right]
$$

where $G_{0}=\frac{2 e^{2}}{h}, \varepsilon_{0}$ represents the level position relative to the fermi level of the electrodes, $\Gamma_{S, D}$ are the coupling parameters of the molecule to the electrodes and are set so that $\Gamma_{S}=\Gamma_{D}$ [42].

To the best of our knowledge, these are the first reported results of BT measured in Au breakjunction devices. The shape of the I-V curves are very consistent with measurements on similar phenyl compounds in mechanically controlled break junctions[43] and first principles calculations[44]. However, there are a number of differences due to the different molecules used in this study. In contrast to previous work that had molecules with two thiols that could bridge on opposite sides, the molecules used in this study can only bind to Au on one side. The large current (10 $\mu \mathrm{A}$ at $1.0 \mathrm{~V})$ exhibited in conjunction with the on/off resonant transport indicates strong conduction between the source and drain electrodes. This current is much larger than that observed for a linear bridged biphenyl dithiol[45], having an experimentally measured conductance value of $1.7 \times 10^{-4} \mathrm{G}_{0}$, which further indicates the molecules are in a different geometry. The strong symmetry in the currentvoltage curves also provides further evidence of a transmission function that is independent of the direction of current flow, as expected in a symmetric system having similar thiol-gold barriers on the drain and source electrodes. We speculate that the high conduction $\left(\approx 0.13 \mathrm{G}_{0}\right.$ at $\left.1.0 \mathrm{~V}\right)$ is due to overlapping $\pi$ orbitals from the stacked benzene rings that are bound from opposing electrodes, as shown in Figure 8 (e). This has similarly been observed in $\pi$-folded molecular junctions[46].

Further information can be gained by examining the data using Transition Voltage Spectroscopy (TVS), which is now a commonly used tool for probing the frontier molecular orbitals (HOMO or LUMO) in molecular devices. First discussed[47] in terms of tunnelling over an energy barrier but now understood to be better interpreted in terms of coherent Landauer[48] transport, TVS studies have been used both experimentally and theoretically to investigate a variety of partially bridged and fully bridged electrode-molecule-electrode systems. Studies are typically based on traditional gold electrodes[49] but also novel approaches such as contacts made with graphene and carbon nanotubes[50-52].

In Figure 9 the $\mathrm{I}-\mathrm{V}$ graphs are analysed by plotting $\ln \left(\mathrm{I} / \mathrm{V}^{2}\right)$ vs $1 / \mathrm{V}$, the so-called Fowler-Nordheim (F-N) plot that is used to study the tunnelling to field-emission transition in solid-state junctions. A key feature of the TVS plot is the transition voltage, $V_{\text {trans, }}$ which is the position of the first minimum and is proportional to the relative difference between the energy of the highest occupied molecular orbital (HOMO) and the Fermi energy. Figure 9 shows a TVS plot for the data of several I-V sweeps shown in Figure 8 (b). From the graph there is a clear minimum at a voltage $9.51 \mathrm{~V}^{-1}$, which gives a transition voltage of $\mathrm{V}_{\text {trans }}=0.11 \mathrm{~V}$.

Although no previous data exists for the system investigated in this article, a comparison of experimental and theoretical values of $V_{\text {trans }}$ with molecules of similar type can be made. In the case of a single phenyl-SH molecule, bonded only to a single gold electrode, a range of theoretical values based on $a b$ initio calculations exist, including $0.32 \mathrm{~V}$ [53], $0.9 \mathrm{~V}$ [54] and $1.69 \mathrm{~V}$ [55]. In terms of experimental measurements, a value of $0.95 \pm 0.11 \mathrm{~V}$ [47] has been found, which would indicate a 
Figure 9. a) Transition Voltage Spectroscopy plot showing a voltage minimum at $9.51 \mathrm{~V}^{-1}$ corresponding to a transition voltage of $V_{\text {trans }}=0.11 \mathrm{~V}$. The transition voltage relates to the difference between the energy of the highest occupied molecular orbital (HOMO) and the Fermi energy of the electrode.

\section{Conclusion}

The fabrication of low cost, high-resolution nanoscale devices has been demonstrated using a fast and high throughput, bilayer nanoimprint lithography approach. The approach, allowing the replication of both nano and micron scale features is applicable to a wide range of device types in nano and microelectronics. Optimum parameters were obtained to achieve highly quality imprinting, speed of imprinting and highly reliable lift-off process after metallization. The flow behaviour of the bilayer resist was discussed in relation to the control of flow defects and preserving the bilayer structure to

midpoint value of the theoretical range is appropriate. In bridged systems, whereby the phenyl molecules have thiols attached to both drain and source electrodes, experimentally determined TVS values of $0.16 \mathrm{~V}$ [56] and $0.79 \pm 0.2 \mathrm{~V}$ [49] have been found for a single phenyl molecule (benzene-1,4dithiol) and for two phenyl molecules (biphenyl-4,4'-dithiol) respectively. Thus from these literature values, our TVS value of $0.11 \mathrm{~V}$ is of closest match to the bridged single phenyl molecule (benzenel), having a TVS minimum at $0.16 \mathrm{~V}$. This comparison suggests the TVS value is largely via vertical stacking. We expect this is because of the nature of the $\mathrm{sp}^{2}$ hybridized carbons in the benzene molecule, which forms a ring of delocalized $\pi$ electrons above the plane of the benzene molecule. In the case of two or more stacked aromatics, such a $\pi$-stacked configuration supports through-space transfer which allows the exchange of electrons[57] over sometimes long-range distances[58]. The importance of $\pi-\pi$ overlap has long been recognized in thin-film organic electronics (small molecule and polymeric systems), tailored graphene devices[59], supramolecular chemistry and electron transport in biological systems. It has also been used to guide the formation of molecular bridges[60] in single molecule electronics.

In both cases, we expect the low value of the TVS is due to the close alignment between the HOMO level (transport through the HOMO level is known to dominate in this system[61]) of the molecule and the electrodes which is hybridized because of the strong coupling between the thiol and electrode. This is particularly applicable in the case of small molecules[42].

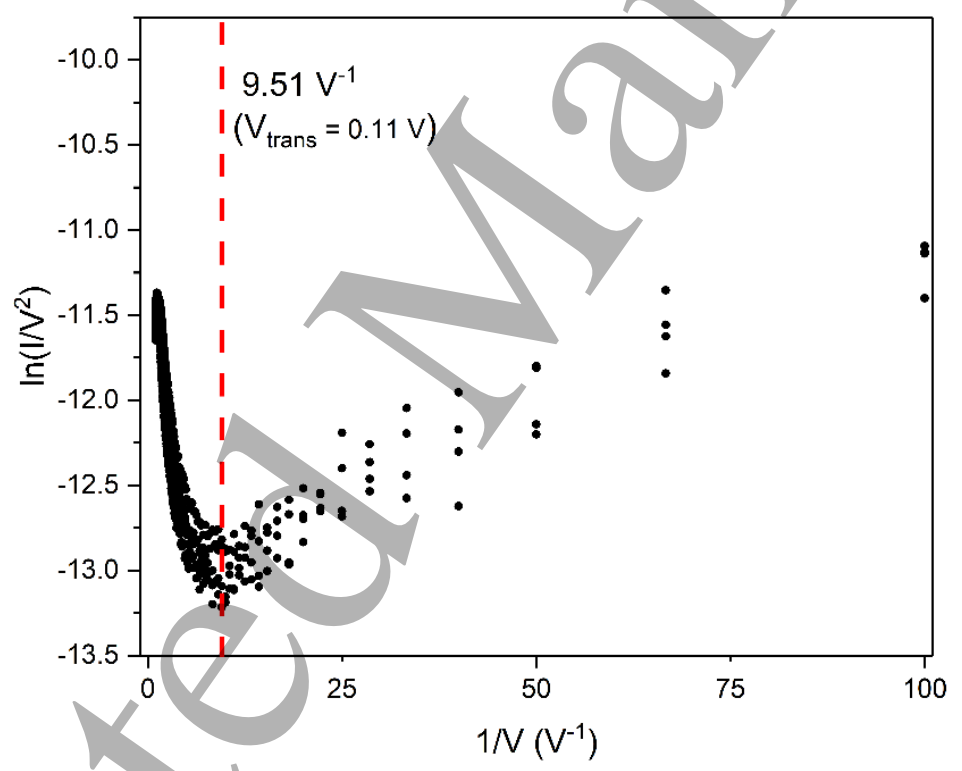


enable successful tear-free lift-off after metal deposition. The optimum imprinting speed and replication of the features was obtained at a temperature of $180^{\circ} \mathrm{C}$ and pressure of 2 bar. The bilayer resist structure is maintained after imprinting allowing the development of an undercut and successful lift-off of a thermally deposited $20 \mathrm{~nm}$ gold thin film without the need for ultra-sonication. Following fabrication of the nanoscale devices by the bilayer nanoimprint approach, feedback controlled electromigration was used to make nanogaps with separation between the electrodes of less than 2 $\mathrm{nm}$, as determined by fitting of the I-V tunnelling curves to the Simmons tunnel model.

This high throughput method of device fabrication has promising applications in the field of molecular electronics as it allows a large number of devices to be fabricated in one-step, at low cost, and with high speed. To demonstrate the applicability of this approach, devices were made having electrodes functionalized with benzenethiol molecules so that the molecules bridged across the gap to form a coupled junction, as confirmed by the observation of molecular transport signatures in repeated I-V sweeps. In this case, transport through molecular levels is indicated by a current suppression present in the I-V behaviour at low voltage, which is then lifted at high voltage, signifying on- and off-resonant transport as a function of voltage. The large conductance, symmetry of the $\mathrm{I}-\mathrm{V}$ sweep and small value of the voltage minimum in Transition Voltage Spectroscopy, $V_{\text {trans }}=0.11 \mathrm{~V}$, indicates the bridging of the two benzenethiol molecules is by $\pi$-stacking.

Formation of the electrodes in this way, having bridged benzenethiol molecules, could have important application for the development of new types of electronic switches/memory devices. Here the device resistance is dependent upon the electronic coupling between the two benzenethiol molecules. This coupling would be dependent upon both the conformation and or electronic spin state of a small molecule present within the transport pathway, which has the ability to be modulated by an external field.

\section{Acknowledgments}

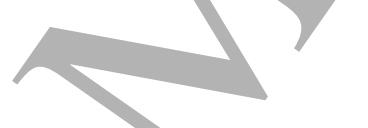

We would like to thank Garry S Robinson and Tony Sinclair for help acquiring SEM images and also the members of Aalto Nanospin group for help with stamp fabrication. The authors would like to acknowledge the contribution of COST action CA15128 (MolSpin) to this work.

\section{$\underline{\text { References }}$}

[1] Aviram A and Ratner M A 1974 Molecular Rectifiers Chem. Phys. Lett. 29 277-83

[2] Reecht G, Scheurer F, Speisser V, Dappe Y J, Mathevet F and Schull G 2014 Electroluminescence of a polythiophene molecular wire suspended between a metallic surface and the tip of a scanning tunneling microscope Phys. Rev. Lett. 112 1-5

[3] Capozzi B, Xia J, Adak O, Dell E J, Liu Z F, Taylor J C, Neaton J B, Campos L M and Venkataraman $L 2015$ Single-molecule diodes with high rectification ratios through environmental control Nat. Nanotechnol. 10 522-7

[4] Thiele S, Balestro F, Ballou R, Klyatskaya S, Ruben M and Wernsdorfer W 2014 Electrically driven nuclear spin resonance in single-molecule magnets Science 344 1135-8

[5] Xiang D, Jeong H, Lee T and Mayer D 2013 Mechanically controllable break junctions for molecular electronics Adv. Mater. 25 4845-67 
[6] Xiang D, Wang X, Jia C, Lee T and Guo X 2016 Molecular-Scale Electronics: From Concept to Function Chem. Rev. 1164318

[7] Kemp N T, Newbury R, Cochrane J W and Dujardin E 2011 Electronic transport in conducting polymer nanowire array devices. Nanotechnology 22105202

[8] Lunc-Popa P, Dalmas G, Faramarzi V, Dayen J F, Majjad H, Kemp N T and Doudin B 2011 Heteronanojunctions with atomic size control using a lab-on-chip electrochemical approach with integrated microfluidics Nanotechnology 22

[9] Popa P L, Kemp N T, Majjad H, Dalmas G, Faramarzi V, Andreas C, Hertel R and Doudin B 2014 The magnetoelectrochemical switch Proc. Natl. Acad. Sci. U. S. A. 111 10433-7

[10] Waldrop M M 2016 More than Moore Nature 530 144-7

[11] Cuevas J C and Scheer E 2015 Molecular Electronics: An Introduction to Theory and Experiment vol 1, ed M Reed (World Scientific)

[12] Gkoupidenis P, Koutsouras D A and Malliaras G G 2017 Neuromorphic device architectures with global connectivity through electrolyte gating Nat. Commun. 815448

[13] Jaafar A H, Gray R J, Verrelli E, O’Neill M, Kelly S M and Kemp N T 2017 Reversible optical switching memristors with tunable STDP synaptic plasticity: A route to hierarchical control in artificial intelligent systems Nanoscale 9 17091-8

[14] Higashiki T, Nakasugi T and Yoneda I 2011 Nanoimprint lithography for semiconductor devices and future patterning innovation Altern. Lithogr. Technol. III 7970797003

[15] Wachenschwanz D, Roddick E, Homola A, Dorsey P, Harper B, Treves D and Bajorek C 2005 Design of a manufacturable discrete track recording medium IEEE Trans. Magn. 41 670-5

[16] Ahn S W, Lee K D, Kim J S, Kim S H, Lee S H, Park J Do and Yoon P W 2005 Fabrication of subwavelength aluminum wire grating using nanoimprint lithography and reactive ion etching Microelectron. Eng. 78-79 314-8

[17] Cardinale G F, Skinner J L, Talin A A, Brocato R W, Palmer D W, Mancini D P, Dauksher W J, Gehoski K, Le N, Nordquist K J and Resnick D J 2004 Fabrication of a surface acoustic wavebased correlator using step-and-flash imprint lithography J. Vac. Sci. Technol. B Microelectron. Nanom. Struct. 223265

[18] Chou S Y, Krauss P R and Renstrom P J 1996 Nanoimprint lithography J. Vac. Sci. Technol. B Microelectron. Nanom. Struct. 144129

[19] Jiang Y, Luo B and Cheng X 2019 Enhanced Thermal Stability of Thermoplastic Polymer Nanostructures for Nanoimprint Lithography Materials (Basel). 12545

[20] Faircloth B, Rohrs H, Tiberio R, Ruoff R and Louis S 2000 Bilayer, nanoimprint lithography J. Vac. Sci. Technol. B Microelectron. Nanom. Struct. $181866-73$

[21] Carlberg P, Graczyk M, Sarwe E, Maximov I, Beck M and Montelius L 2003 Lift-off process for nanoimprint lithography Microelectron. Eng. 68 203-7

[22] Guo L J 2007 Nanoimprint lithography: Methods and material requirements Adv. Mater. 19 495-513

[23] Beaufrand J-B, Dayen J-F, Kemp N T, Sokolov A and Doudin B 2011 Magnetoresistance signature of resonant states in electromigrated Ni nanocontacts Appl. Phys. Lett. 98142504

[24] Wu Z M, Steinacher M, Huber R, Calame M, Van Der Molen S J and Schönenberger C 2007 
Feedback controlled electromigration in four-terminal nanojunctions Appl. Phys. Lett. 91 114

[25] Hadeed F O and Durkan C 2007 Controlled fabrication of 1-2 nm nanogaps by electromigration in gold and gold-palladium nanowires Appl. Phys. Lett. 91 2005-8

[26] Heyderman L., Schift H, David C, Gobrecht J and Schweizer T 2000 Flow behaviour of thin polymer films used for hot embossing lithography Microelectron. Eng. 54 229-45

[27] Scheer $\mathrm{H}$ and Schulz $\mathrm{H} 2001 \mathrm{~A}$ contribution to the flow behaviour of thin polymer films during hot embossing lithography 56 311-32

[28] Park H, Lim A K L, Alivisatos A P, Park J and McEuen P L 1999 Fabrication of metallic electrodes with nanometer separation by electromigration Appl. Phys. Lett. $75301-3$

[29] Doudin B and Kemp N T 2011 Chapter 28: Ballistic Spin Transport Handbook of Spin Transport and Magnetism ed E Y Tsymbal and I Zutic (Chapman and Hall/CRC)

[30] Wu Z M 2008 Formation mechanism and resistance fluctuations of atomic sized junctions (University of Basel)

[31] Heersche H B, Lientschnig G, O'Neill K, Van Der Zant H S J and Zandbergen H W 2007 In situ imaging of electromigration-induced nanogap formation by transmission electron microscopy Appl. Phys. Lett. 91 1-4

[32] Girod S, Bubendorff J L, Montaigne F, Simon L, Lacour D and Hehn M 2012 Real time atomic force microscopy imaging during nanogap formation by electromigration Nanotechnology 23 365302

[33] Van Der Zant H S J, Kervennic Y V, Poot M, O'Neill K, De Groot Z, Thijssen J M, Heersche H B, Stuhr-Hansen N, Bjørnholm T, Vanmaekelbergh D, Van Walree C A and Jenneskens L W 2006 Molecular three-terminal devices: Fabrication and measurements Faraday Discuss. 131 34756

[34] Esen G and Fuhrer M S 2005 Temperature control of electromigration to form gold nanogap junctions Appl. Phys. Lett. 87 1-3

[35] Campbell J M and Knobel R G 2013 Feedback-controlled electromigration for the fabrication of point contacts Appl. Phys. Lett. 102 1-5

[36] Austin M and Chou S Y 2002 Fabrication of nanocontacts for molecular devices using nanoimprint lithography J. Vac. Sci. Technol. B Microelectron. Nanom. Struct. 20 665-7

[37] Simmons J G 1963 Generalized Formula for the Electric Tunnel Effect between Similar Electrodes Separated by a Thin Insulating Film J. Appl. Phys. 34 1793-803

[38] Szafranski C A, Tanner W, Laibinis P E and Garrell R L 1998 Surface-enhanced Raman spectroscopy of aromatic thiols and disulfides on gold electrodes Langmuir 14 3570-9

[39] Nuzzo R G, Zegarski B R and DuBois L H 1987 Fundamental Studies of the Chemisorption of Organosulfur Compounds on Au( 111). Implications for Molecular Self-Assembly on Gold Surfaces J. Am. Chem. Soc. 109 733-40

[40] Huang F K, Horton R C, Myles D C and Garrell R L 2002 Selenolates as Alternatives to Thiolates for Self-Assembled Monolayers: A SERS Study Langmuir 14 4802-8

[41] Ashwell G J, Phillips L J, Robinson B J, Barnes S A, Williams A T, Urasinska-Wojcik B, Lambert C J, Grace I M, Cox T I and Sage I C 2011 Synthesis of covalently linked molecular bridges 
between silicon electrodes in CMOS-based arrays of vertical Si/SiO 2/Si nanogaps Angew. Chemie - Int. Ed. 50 8722-6

[42] Perrin M L, Burzurí E and van der Zant H S J 2015 Single-molecule transistors Chem. Soc. Rev. 44 902-19

[43] Reed M A, Zhou C, Muller C J, Burgin T P and Tour J M 1997 Conductance of a Molecular Junction Science $\mathbf{2 7 8} 252-4$

[44] Ventra M Di, Pantelides S T and Lang N D 2000 First-Principles Calculation of Transport Properties of a Molecular Device Phys. Rev. Lett. 84 979-82

[45] Mishchenko A, Vonlanthen D, Meded V, Bürkle M, Li C, Pobelov I V., Bagrets A, Viljas J K, Pauly F, Evers F, Mayor M and Wandlowski T 2010 Influence of conformation on conductance of biphenyl-dithiol single-molecule contacts Nano Lett. 10 156-63

[46] Carini M, Ruiz M P, Usabiaga I, Fernández J A, Cocinero E J, Melle-Franco M, Diez-Perez I and Mateo-Alonso A 2017 High conductance values in $\pi$-folded molecular junctions Nat. Commun. 8

[47] Beebe J M, Kim B, Gadzuk J W, Frisbie C D and Kushmerick J G 2006 Transition from direct tunneling to field emission in metal-molecule-metal junctions Phys. Rev. Lett. 97 1-4

[48] Huisman E H, Guédon C M, Van Wees B J and Van Der Molen S J,2009 Interpretation of transition voltage spectroscopy Nano Lett. 9 3909-13

[49] Guo S, Hihath J, Díez-Pérez I and Tao N 2011 Measurement and statistical analysis of singlemolecule current-voltage characteristics, transition voltage spectroscopy, and tunneling barrier height J. Am. Chem. Soc. 133 19189-97

[50] Brito Da Silva C A, De Araujo Pinheiro F A and Del Nero J 2016 Organic nano-devices composed by carbon nanotube/oligophenylenes/carbon nanotube junctions: Transitionvoltage spectroscopy, applications and chirality versus geometry J. Nanosci. Nanotechnol. 16 $9771-8$

[51] Brito Silva C A, Da Silva S J S, Granhen E R, Leal J F P, Del Nero J and Pinheiro F A 2010 Electronic transport in biphenyl single-molecule junctions with carbon nanotubes electrodes: The role of molecular conformation and chirality Phys. Rev. B - Condens. Matter Mater. Phys. 82 14-7

[52] Brito Silva C A, Da Silva S J S, Leal J F P, Pinheiro F A and Del Nero J 2011 Electronic transport in oligo-para-phenylene junctions attached to carbon nanotube electrodes: Transitionvoltage spectroscopy and chirality Phys. Rev. B - Condens. Matter Mater. Phys. 83 1-6

[53] Araidai M and Tsukada M 2010 Theoretical calculations of electron transport in molecular junctions: Inflection behavior in Fowler-Nordheim plot and its origin Phys. Rev. B - Condens. Matter Mater. Phys. 81 1-7

[54] Wu K, Bai M, Sanvito S and Hou S 2013 Quantitative interpretation of the transition voltages in gold-poly(phenylene) thiol-gold molecular junctions J. Chem. Phys. 139194703

[55] Chen J, Markussen T and Thygesen K S 2010 Quantifying transition voltage spectroscopy of molecular junctions: Ab initio calculations Phys. Rev. B - Condens. Matter Mater. Phys. 82 1-4

[56] Bruot C, Hihath J and Tao N 2012 Mechanically controlled molecular orbital alignment in single molecule junctions Nat. Nanotechnol. 7 35-40

[57] Schneebeli S T, Kamenetska M, Cheng Z, Skouta R, Friesner R A, Venkataraman L and Breslow 
R 2011 Single-molecule conductance through multiple $\pi-\pi$-stacked benzene rings determined with direct electrode-to-benzene ring connections J. Am. Chem. Soc. 133 2136-9

[58] Slinker J D, Muren N B, Renfrew S E and Barton J K 2011 DNA charge transport over 34 nm. Nat. Chem. 3 228-33

[59] Zhang Z, Huang H, Yang X and Zang L 2011 Tailoring electronic properties of graphene by $\pi-\pi$ Stacking with aromatic molecules J. Phys. Chem. Lett. 2 2897-905

[60] Wu S, González M T, Huber R, Grunder S, Mayor M, Schönenberger C and Calame M 2008 Molecular junctions based on aromatic coupling Nat. Nanotechnol. 3 569-74

[61] Song H, Kim Y, Jang Y H, Jeong H, Reed M A and Lee T 2009 Observation of /molecular orbital gating Nature 462 1039-43 